\title{
Further evidence for the non-existence of a unified hoop conjecture
}

\author{
Shahar Hod ${ }^{1,2, a}$ \\ ${ }^{1}$ The Ruppin Academic Center, 40250 Emek Hefer, Israel \\ 2 The Hadassah Academic College, 91010 Jerusalem, Israel
}

Received: 24 July 2020 / Accepted: 14 October 2020 / Published online: 22 October 2020

(C) The Author(s) 2020

\begin{abstract}
The hoop conjecture, introduced by Thorne almost five decades ago, asserts that black holes are characterized by the mass-to-circumference relation $4 \pi \mathcal{M} / \mathcal{C} \geq 1$, whereas horizonless compact objects are characterized by the opposite inequality $4 \pi \mathcal{M} / \mathcal{C}<1$ (here $\mathcal{C}$ is the circumference of the smallest ring that can engulf the self-gravitating compact object in all azimuthal directions). It has recently been proved that a necessary condition for the validity of this conjecture in horizonless spacetimes of spatially regular charged compact objects is that the mass $\mathcal{M}$ be interpreted as the mass contained within the engulfing sphere (and not as the asymptotically measured total ADM mass). In the present paper we raise the following physically intriguing question: is it possible to formulate a unified version of the hoop conjecture which is valid for both black holes and horizonless compact objects? In order to address this important question, we analyze the behavior of the mass-to-circumference ratio of Kerr-Newman black holes. We explicitly prove that if the mass $\mathcal{M}$ in the hoop relation is interpreted as the quasilocal Einstein-Landau-Lifshitz-Papapetrou and Weinberg mass contained within the black-hole horizon, then these charged and spinning black holes are characterized by the $s u b$-critical mass-to-circumference ratio $4 \pi \mathcal{M} / \mathcal{C}<1$. Our results provide evidence for the non-existence of a unified version of the hoop conjecture which is valid for both black-hole spacetimes and spatially regular horizonless compact objects.
\end{abstract}

\section{Introduction}

The influential hoop conjecture has been suggested by Thorne [1] as a simple necessary and sufficient condition for the formation of black holes in dynamical gravitational collapse scenarios. In particular, the hoop criterion asserts that a self-gravitating matter configuration of mass $\mathcal{M}$ would collapse to form a black hole if and only if a circular hoop of a

\footnotetext{
a e-mail: shaharhod@gmail.com (corresponding author)
}

critical circumference $\mathcal{C}_{\text {critical }}=4 \pi \mathcal{M}$ can be placed around the self-gravitating matter distribution and rotated in $360^{\circ}$ to form an engulfing sphere. The hoop conjecture therefore implies the simple relation [1]

$\mathcal{H} \equiv \frac{4 \pi \mathcal{M}}{\mathcal{C}} \geq 1 \Longleftrightarrow$ Black-hole horizon exists.

In his original formulation of the hoop conjecture, Thorne [1] has not provided an explicit definition for the mass term $\mathcal{M}$ in his dimensionless mass-to-circumference ratio $\mathcal{H} \equiv 4 \pi \mathcal{M} / \mathcal{C}$ [2]. Interestingly, as explicitly demonstrated in [3-5], the hoop conjecture (1) can be violated in curved spacetimes of horizonless charged compact objects if $\mathcal{M}$ is interpreted as the total ADM mass of the spacetime. In particular, as nicely shown in [5], spherically symmetric horizonless thin shells of radius $R$, electric charge $Q$, and total ADM mass $M$ can be characterized by the dimensionless relation $M / R \rightarrow 1^{-}$in the $Q / M \rightarrow 1^{-}$limit. Thus, these horizonless charged shells are characterized by the super-critical dimensionless relation $4 \pi M / \mathcal{C} \rightarrow 2^{-}$and can therefore violate the hoop conjecture (1) if $\mathcal{M}$ is interpreted as the total ADM mass $M$ of the spacetime.

Intriguingly, it has recently been proved [6] (see also [7]) that if the mass $\mathcal{M}$ is interpreted as the gravitating mass $M_{\text {in }} \equiv M(R)$ contained within an engulfing sphere of radius $R$ [8] (and not as the total ADM mass of the spacetime), then horizonless self-gravitating charged compact objects are characterized by the sub-critical dimensionless relation $\mathcal{H}<$ 1 and therefore respect the hoop conjecture (1), see also the physically interesting related works [9-13].

The interesting physical results presented in [3-7] indicate that if there is any hope to formulate the hoop conjecture (1) in a unified way, which is valid for both black-hole spacetimes and spatially regular (horizonless) self-gravitating compact objects, then the mass term $\mathcal{M}$ should not be interpreted as the total ADM mass of the spacetime. In particular, if a valid and unified formulation of the conjecture (1) do exists, then the results presented in [3-7] indicate that the mass $\mathcal{M}$ 
should be interpreted as the mass $M_{\text {in }}$ contained within the boundaries of the compact object (a black hole or a selfgravitating horizonless object).

Motivated by the intriguing results of [3-7], in the present compact paper we raise the following physically interesting question: Is it possible to formulate the hoop conjecture (1) in a unified way which is valid for both black-hole spacetimes and spatially regular horizonless compact objects?

In order to address this intriguing question, we shall analyze the functional behavior of the dimensionless mass-tocircumference ratio $\mathcal{H}(Q, a) \equiv 4 \pi \mathcal{M} / \mathcal{C}$ of charged and spinning Kerr-Newman black-hole spacetimes (here $Q$ and $a$ are respectively the electric charge and the angular momentum per unit mass of the Kerr-Newman black holes). Interestingly, we shall explicitly show below that if the mass $\mathcal{M}$ in the hoop relation (1) is interpreted as the quasilocal Einstein-Landau-Lifshitz-Papapetrou and Weinberg mass $M_{\text {in }}$ contained within the black-hole horizon, then charged and spinning Kerr-Newman black holes are characterized by the sub-critical mass-to-circumference ratio $4 \pi M_{\text {in }} / \mathcal{C}<1$ and therefore violate the hoop conjecture (1).

\section{The hoop conjecture in charged and spinning Kerr-Newman black-hole spacetimes}

A Kerr-Newman black-hole spacetime of asymptotically measured ADM mass $M$, electric charge $Q$ [14], and angular momentum $J=M a$ is described by the curved line element $[15-18]$

$$
\begin{aligned}
d s^{2}= & -\frac{\Delta-a^{2} \sin ^{2} \theta}{\rho^{2}} d t^{2}+\frac{\rho^{2}}{\Delta} d r^{2} \\
& -\frac{2 a \sin ^{2} \theta\left(2 M r-Q^{2}\right)}{\rho^{2}} d t d \phi+\rho^{2} d \theta^{2} \\
& +\frac{\left(r^{2}+a^{2}\right)^{2}-a^{2} \Delta \sin ^{2} \theta}{\rho^{2}} \sin ^{2} \theta d \phi^{2},
\end{aligned}
$$

where

$\Delta \equiv r^{2}-2 M r+Q^{2}+a^{2} ; \quad \rho^{2} \equiv r^{2}+a^{2} \cos ^{2} \theta$.

The radii

$r_{ \pm}=M \pm\left(M^{2}-Q^{2}-a^{2}\right)^{1 / 2}$

of the (outer and inner) black-hole horizons are determined by the zeros of the metric function $\Delta(r)$.

Substituting $d t=d r=d \theta=0, \theta=\pi / 2$, and $\Delta \phi=$ $2 \pi$ into the curved line element (2), one finds the simple functional expression

$\mathcal{C}_{\mathrm{eq}}=2 \pi \frac{r_{+}^{2}+a^{2}}{r_{+}} \cdot[1+O(\epsilon)]$ for the equatorial circumference of an engulfing ring which is located at a fixed radial coordinate $r=r_{+} \cdot[1+O(\epsilon)]$ just outside $(\epsilon \ll 1)$ the outer horizon $(4)$ of the KerrNewman black-hole [19]. Taking cognizance of Eq. (4), one can express the black-hole equatorial circumference (5) in the dimensionless form [20]

$\overline{\mathcal{C}}_{\mathrm{eq}}(\bar{Q}, \bar{a})=4 \pi \cdot\left[1-\frac{\bar{Q}^{2}}{2\left(1+\sqrt{1-\bar{Q}^{2}-\bar{a}^{2}}\right)}\right]$,

where

$\overline{\mathcal{C}} \equiv \frac{\mathcal{C}}{M} ; \quad \bar{Q} \equiv \frac{Q}{M} ; \quad \bar{a} \equiv \frac{a}{M}$

are respectively the dimensionless circumference, the dimensionless electric charge, and the dimensionless angular momentum of the Kerr-Newman black hole.

Before proceeding, it is worth pointing out that the hoop relation (1) is respected by all Kerr-Newman black holes if the mass term $\mathcal{M}$ is interpreted as the total ADM mass $M$ of the spacetime. In particular, taking cognizance of Eqs. (6) and (7), one finds the simple dimensionless relation

$\mathcal{H}(\bar{Q}, \bar{a}) \equiv \frac{4 \pi M_{\mathrm{tot}}}{\mathcal{C}}=\left[1-\frac{\bar{Q}^{2}}{2\left(1+\sqrt{1-\bar{Q}^{2}-\bar{a}^{2}}\right)}\right]^{-1} \geq 1$

for the charged and spinning Kerr-Newman black holes.

However, as emphasized above, it has been demonstrated explicitly in [3-5] that the hoop conjecture (1) can be violated by spatially regular horizonless charged objects if the mass $\mathcal{M}$ is interpreted as the total ADM mass of the spacetime. This fact implies that a unified version of the hoop conjecture, which should be valid for both black-hole spacetimes and horizonless compact objects, cannot be formulated in terms of the total ADM mass of the spacetime.

On the other hand, it has been explicitly proved in $[6,7]$ that the hoop relation (1) is respected by horizonless charged compact objects if the mass term $\mathcal{M}$ is interpreted as the mass $M_{\text {in }}(R)$ contained within the engulfing sphere of radius $R$. This observation indicates that if a unified formulation of the hoop conjecture (1) exists, then the mass $\mathcal{M}$ should be interpreted as the mass contained within the engulfing sphere [8].

We shall therefore test the validity of the hoop relation (1) for Kerr-Newman black holes with the mass term $\mathcal{M}$ interpreted as the mass $M_{\text {in }}\left(r_{+}\right)$contained within the black-hole horizon. To this end, we first note that, in a physically interesting paper, Aguirregabiria, Chamorro, and Virbhadra [21] have analyzed the energy distribution in the Kerr-Newman black-hole spacetime using various physical prescriptions (namely, the Einstein, the Landau-Lifshitz, the Papapetrou, 
and the Weinberg prescriptions [21]) for calculating energy densities in general relativity. Interestingly, it has been explicitly shown in [21] that the Einstein, Landau-Lifshitz, Papapetrou, and Weinberg prescriptions provide the same compact analytical expression [21,22]

$M_{\mathrm{in}} \equiv M_{\mathrm{in}}\left(r=r_{+}\right)=M-\frac{Q^{2}}{4 r_{+}}\left[1+\frac{r_{+}^{2}+a^{2}}{a r_{+}} \cdot \arctan \left(\frac{a}{r_{+}}\right)\right]$

for the quasilocal Einstein-Landau-Lifshitz-Papapetrou and Weinberg mass $M_{\text {in }}$ contained within the horizon of a KerrNewman black hole. Taking cognizance of Eqs. (4) and (9), one can express the engulfed gravitational mass $M_{\mathrm{in}}$ of the charged and spinning Kerr-Newman black hole (2) explicitly in terms of its dimensionless physical parameters $\bar{Q}$ and $\bar{a}$ :

$$
\begin{aligned}
\bar{M}_{\text {in }}(\bar{Q}, \bar{a})= & 1-\frac{\bar{Q}^{2}}{4\left(1+\sqrt{1-\bar{Q}^{2}-\bar{a}^{2}}\right)} \\
& \times\left[1+\frac{\left(1+\sqrt{1-\bar{Q}^{2}-\bar{a}^{2}}\right)^{2}+\bar{a}^{2}}{\bar{a}\left(1+\sqrt{1-\bar{Q}^{2}-\bar{a}^{2}}\right)}\right. \\
& \left.\cdot \arctan \left(\frac{\bar{a}}{1+\sqrt{1-\bar{Q}^{2}-\bar{a}^{2}}}\right)\right],
\end{aligned}
$$

where

$\bar{M}_{\text {in }} \equiv \frac{M_{\text {in }}}{M}$.

In Table 1 we present the dimensionless mass-to-circumference ratio [see Eqs. (6) and (10)]

$\mathcal{H}(\bar{Q}, \bar{a}) \equiv \frac{4 \pi \mathcal{M}}{\mathcal{C}_{\mathrm{eq}}} ; \quad \mathcal{M}=M_{\mathrm{in}}\left(r_{+}\right)$

of charged and spinning Kerr-Newman black holes for various values of the black-hole dimensionless physical parameters $\bar{Q}$ and $\bar{a}$. Intriguingly, the data presented in Table 1 reveals the fact that all charged and spinning $(\bar{Q} \neq 0, \bar{a} \neq 0)$ Kerr-Newman black holes are characterized by the subcritical mass-to-circumference relation [23]

$\mathcal{H}(\bar{Q}, \bar{a})<1$.

In particular, from the data presented in Table 1 one finds that, for a given value of the black-hole electric charge $\bar{Q}$, the dimensionless ratio $\mathcal{H}$ is a monotonically decreasing function of the black-hole angular momentum parameter $\bar{a}$. In addition, one finds that, for a given value of the black-hole angular momentum $\bar{a}$, the mass-to-circumference ratio $\mathcal{H}$ is a monotonically decreasing function of the black-hole electric charge $\bar{Q}$. These facts indicate that the dimensionless mass-to-circumference function $\mathcal{H}(\bar{Q}, \bar{a})$ is minimized by an extremal black hole. Substituting $\bar{Q}^{2}+\bar{a}^{2}=1$ for extremal Kerr-Newman black holes [24] into Eqs. (6) and (10), one obtains the compact functional expression [see Eq. (12)]

$\mathcal{H}^{\mathrm{ext}}(\bar{a})=\frac{1-\frac{1-\bar{a}^{2}}{4}\left[1+\frac{1+\bar{a}^{2}}{\bar{a}} \cdot \arctan (\bar{a})\right]}{1-\frac{1-\bar{a}^{2}}{2}}$

for the mass-to-circumference ratio of extremal Kerr-Newman black holes. From (14) one finds

$\min _{\bar{Q}, \bar{a}}\{\mathcal{H}\}=0.9468$ for $(\bar{Q}, \bar{a})=(0.7776,0.6287)$.

\section{Summary and discussion}

The Thorne hoop conjecture [1] serves as a boundary between black-hole configurations and horizonless compact objects. In particular, this physically influential conjecture asserts that black holes should be characterized by the massto-circumference inequality $4 \pi \mathcal{M} / \mathcal{C} \geq 1$, whereas horizonless compact objects should be characterized by the opposite inequality $4 \pi \mathcal{M} / \mathcal{C}<1$.

The physical meaning of the mass term $\mathcal{M}$ in the hoop conjecture (1) has not been specified by Thorne [1]. However, it is known that the hoop relation (1) can be violated by self-gravitating horizonless charged objects if the mass $\mathcal{M}$ is interpreted as the total ADM mass of the spacetime [35]. On the other hand, it has recently been proved $[6,7]$ that spherically symmetric horizonless charged objects respect the hoop relation (1) if the mass $\mathcal{M}$ is interpreted as the gravitating mass $M_{\text {in }} \equiv M(R)$ contained within an engulfing sphere of radius $R$ (and not as the total mass of the spacetime).

The physical results presented in [3-7] imply that a unified version of the hoop relation (which would be valid for both black-hole spacetimes and spatially regular horizonless compact objects), if it exists, should be formulated in terms of the mass $M_{\text {in }}(R)$ contained within the boundaries of the engulfing sphere [8].

Motivated by the interesting physical results of [3-7], in the present compact paper we have raised the following physically intriguing question: Is it possible to formulate a unified version of the hoop conjecture which is valid for both blackhole spacetimes and spatially regular horizonless compact objects?

In order to address this physically important question, we have analyzed the behavior of the dimensionless mass-tocircumference ratio $\mathcal{H}(\bar{Q}, \bar{a}) \equiv 4 \pi \mathcal{M} / \mathcal{C}$ of charged and spinning Kerr-Newman black holes, where $\mathcal{M}=M_{\text {in }}\left(r_{+}\right)$ is the quasilocal Einstein-Landau-Lifshitz-Papapetrou and Weinberg mass contained within the black-hole horizon. The 
Table 1 The dimensionless mass-to-circumference ratio $\mathcal{H}(\bar{Q}, \bar{a}) \equiv$ $4 \pi \mathcal{M} / \mathcal{C}_{\text {eq }}$ of charged and spinning Kerr-Newman black holes. Here $\mathcal{M}$ is interpreted as the quasilocal Einstein-Landau-Lifshitz-Papapetrou and Weinberg mass contained within the black-hole horizon and $\mathcal{C}_{\mathrm{eq}}$ is the equatorial circumference along the black-hole outer horizon [see

\begin{tabular}{llllll}
\hline $\mathcal{H}(\bar{Q}, \bar{a})$ & $\bar{a}=0.0$ & $\bar{a}=0.2$ & $\bar{a}=0.4$ & $\bar{a}=0.6$ & $\bar{a}=0.8$ \\
\hline $\bar{Q}=0.0$ & 1.0000 & 1.0000 & 1.0000 & 1.0000 & 1.0000 \\
$\bar{Q}=0.2$ & 1.0000 & 0.9999 & 0.9998 & 0.9996 & 0.9989 \\
$\bar{Q}=0.4$ & 1.0000 & 0.9998 & 0.9993 & 0.9980 & 0.9944 \\
$\bar{Q}=0.6$ & 1.0000 & 0.9995 & 0.9978 & 0.9934 & 0.9579 \\
$\bar{Q}=0.8$ & 1.0000 & 0.9986 & 0.9929 & 0.9471 & - \\
$\bar{Q}=1.0$ & 1.0000 & - & - & - & - \\
\hline
\end{tabular}

Eqs. (6) and (10)]. Interestingly, the dimensionless ratio $\mathcal{H}(\bar{Q}, \bar{a})$ is found to be a monotonically decreasing function of the black-hole physical parameters $\bar{a}$ and $\bar{Q}$. In particular, one finds that charged and spinning Kerr-Newman black holes are characterized by the $s u b$-critical mass-to-circumference ratio $\mathcal{H}(\bar{Q} \neq 0, \bar{a} \neq 0) \equiv 4 \pi \mathcal{M} / \mathcal{C}_{\text {eq }}<1$ main results derived in this paper and their physical implications are as follows:

(1) While it is well known that neutral spinning Kerr black holes and spherically symmetric charged ReissnerNordström $(\mathrm{RN})$ black holes saturate the hoop relation [see Eqs. (4), (6), and (10) with $\bar{Q}=0$ or $\bar{a}=0$ ]

$$
\frac{4 \pi M_{\mathrm{in}}}{\mathcal{C}_{\mathrm{eq}}}=1 \text { for Kerr and RN black holes, }
$$

we have explicitly proved that charged-spinning $(\bar{Q} \neq$ $0, \bar{a} \neq 0$ ) Kerr-Newman black holes are characterized by the sub-critical dimensionless mass-to-circumference ratio (see the data presented in Table 1) [25-27]

$$
\begin{aligned}
& \frac{4 \pi M_{\text {in }}}{\mathcal{C}_{\mathrm{eq}}}<1 \text { for charged and } \\
& \text { spinning Kerr-Newman black holes. }
\end{aligned}
$$

(2) It has been shown that, for Kerr-Newman black holes, the dimensionless mass-to-circumference ratio $\mathcal{H}(\bar{Q}, \bar{a}) \equiv$ $4 \pi M_{\text {in }} / \mathcal{C}_{\text {eq }}$ is a monotonically decreasing function of the black-hole physical parameters $\bar{Q}$ and $\bar{a}$. In particular, for a given value $\bar{Q}$ of the black-hole electric charge, the hoop relation $\mathcal{H}(\bar{a} ; \bar{Q})$ is minimized by the extremal Kerr-Newman black hole with $\bar{a}=\sqrt{1-\bar{Q}^{2}}$. Likewise, for a given value $\bar{a}$ of the black-hole angular momentum parameter, the hoop relation $\mathcal{H}(\bar{Q} ; \bar{a})$ is minimized by the extremal Kerr-Newman black hole with $\bar{Q}=\sqrt{1-\bar{a}^{2}}$.

(3) It has been found that the generic violations of the hoop conjecture (1) with $\mathcal{M}=M_{\text {in }}\left(r_{+}\right)$by the Kerr-Newman family of charged and spinning black holes are always bounded from below by the relation $0.9468 \lesssim \mathcal{H} \equiv$ $4 \pi M_{\text {in }} / \mathcal{C}_{\text {eq }} \leq 1$ [see Eq. (15)] [28].

(4) The results presented in this paper provide compelling evidence for the non-existence of a unified version of the hoop conjecture which is valid for both black-hole spacetimes and spatially regular horizonless compact objects. In particular, if the mass term $\mathcal{M}$ in the hoop conjecture is interpreted as the total ADM mass of the spacetime then, as explicitly proved in [3-7], horizonless charged compact objects can violate the hoop relation (1). On the other hand, in the present paper we have explicitly proved that if the mass term $\mathcal{M}$ in the hoop conjecture is interpreted as the quasilocal EinsteinLandau-Lifshitz-Papapetrou and Weinberg mass contained within the engulfing sphere, then charged and spinning Kerr-Newman black holes violate the hoop relation (1).

Acknowledgements This research is supported by the Carmel Science Foundation. I would like to thank Yael Oren, Arbel M. Ongo, Ayelet B. Lata, and Alona B. Tea for stimulating discussions.

Data Availability Statement This manuscript has no associated data or the data will not be deposited. [Authors' comment: All data generated or analysed during this study are included in this published article.]

Open Access This article is licensed under a Creative Commons Attribution 4.0 International License, which permits use, sharing, adaptation, distribution and reproduction in any medium or format, as long as you give appropriate credit to the original author(s) and the source, provide a link to the Creative Commons licence, and indicate if changes were made. The images or other third party material in this article are included in the article's Creative Commons licence, unless indicated otherwise in a credit line to the material. If material is not included in the article's Creative Commons licence and your intended use is not permitted by statutory regulation or exceeds the permitted use, you will need to obtain permission directly from the copyright holder. To view a copy of this licence, visit http://creativecomm ons.org/licenses/by/4.0/.

Funded by $\mathrm{SCOAP}^{3}$.

\section{References}

1. K.S. Thorne, in Magic without Magic: John Archibald Wheeler, ed. by J. Klauder (Freeman, San Francisco, 1972)

2. We use natural units in which $G=c=\hbar=1$. In these units the fundamental ratio $\mathcal{H} \equiv 4 \pi \mathcal{M} / \mathcal{C}$ is dimensionless 
3. J.P. de León, Gen. Relativ. Gravit. 19, 289 (1987)

4. W.B. Bonnor, Phys. Lett. A 99, 424 (1983)

5. H. Andréasson, Commun. Math. Phys. 288, 715 (2009)

6. S. Hod, Eur. Phys. J. C 78, 1013 (2018). arXiv:1903.09786

7. Y. Peng, Eur. Phys. J. C 79, 943 (2019)

8. It is worth emphasizing that the engulfing sphere is formed from the rotation of the engulfing ring by $360^{\circ}$

9. P. Bizoń, E. Malec, N. O’Murchadha, Phys. Rev. Lett. 61, 1147 (1988)

10. P. Bizoń, E. Malec, N. O’Murchadha, Class. Quantum Gravity 6, 961 (1989)

11. E. Malec, Phys. Rev. Lett. 67, 949 (1991)

12. E. Malec, Acta Phys. Pol. B 22, 829 (1991)

13. M. Iriondo, E. Malec, N. O' Murchadha, Phys. Rev. D 54, 4792 (1996)

14. We shall assume $Q \geq 0$ without loss of generality

15. J.M. Bardeen, W.H. Press, S.A. Teukolsky, Astrophys. J. 178, 347 (1972)

16. S. Chandrasekhar, The Mathematical Theory of Black Holes (Oxford University Press, New York, 1983)

17. S.L. Shapiro, S.A. Teukolsky, Black Holes, White Dwarfs, and Neutron Stars: The Physics of Compact Objects (Wiley, New York, 1983)

18. We use here the familiar Boyer-Lindquist spacetime coordinates $(t, r, \theta, \phi)[15-17]$

19. It is important to note that, due to the rotation of the Kerr-Newman black holes, the circumference $\mathcal{C}_{\mathrm{p}}$ of an engulfing polar hoop, which is perpendicular to the equatorial plane of the rotating black hole, is smaller than the circumference (5) of the equatorial engulfing hoop: $\mathcal{C}_{\mathrm{p}}<\mathcal{C}_{\text {eq }}$. This physically important property of the spinning KerrNewman black-hole spacetimes can be deduced mathematically by substituting $d t=d r=d \phi=0$ and $\Delta \theta=2 \pi$ into the curved line element (2) and using the characteristic inequalities [see Eq. (3)] $\rho^{2} \leq r^{2}+a^{2}<r^{2}+a^{2}+2 M a^{2} / r$ for the spinning $(a \neq 0)$ black holes

20. It is interesting to note that one finds from Eq. (6) that the dimensionless equatorial circumference $\overline{\mathcal{C}}_{\text {eq }}(\bar{Q}=0, \bar{a})$ of a neutral Kerr black hole is universal in the sense that it is independent of its dimensionless angular momentum $\bar{a}$

21. J.M. Aguirregabiria, A. Chamorro, K.S. Virbhadra, Gen. Relativ. Gravit. 28, 1393 (1996)
22. Note that for non-rotating $(a \rightarrow 0)$ black holes, one finds from (9) the simple relation $M_{\text {in }}=M-Q^{2} / 2 r_{+}$

23. Note that spherically symmetric black holes are characterized by the simple relation $M_{\text {in }}\left(r_{+}\right)=\frac{1}{2} r_{+}$for the mass contained within the black-hole horizon. Thus, if the mass term $\mathcal{C}$ in the hoop conjecture (1) is interpreted as the mass $M_{\text {in }}\left(r_{+}\right)$contained within the black-hole horizon (and not as the total ADM mass of the spacetime), then all spherically symmetric black holes would trivially saturate the hoop relation (1)

24. Note that extremal Kerr-Newman black holes are characterized by the simple relation $r_{-}=r_{+}$[see Eq. (4)]

25. It is worth noting that the Komar [26] and Møller [27] prescriptions for calculating energy densities in general relativity yield the expression $M_{\mathrm{in}} \equiv M\left(r=r_{+}\right)=M-\frac{Q^{2}}{2 r_{+}}\left[1+\frac{r_{+}^{2}+a^{2}}{a r_{+}} \cdot \arctan \left(\frac{a}{r_{+}}\right)\right]$ for the mass $M_{\text {in }}$ contained within the horizon of a Kerr-Newman black hole. This expression for $M_{\text {in }}$ is smaller than the corresponding expression (9) for the mass contained within the black-hole horizon according to the Einstein, the Landau-Lifshitz, the Papapetrou, and the Weinberg prescriptions for calculating energy densities in general relativity [21]. Thus, according to the Komar and Møller prescriptions for calculating the mass contained within the black-hole horizon, Kerr-Newman black-hole spacetimes are characterized by smaller mass-to-circumference ratios than the ones presented in Table 1. This fact implies, in particular, that if one identifies the mass $\mathcal{M}$ in the hoop relation (1) as the mass $M_{\text {in }}$ contained within the black-hole horizon according to the Komar and Møller prescriptions [26,27], then the violations of the hoop conjecture (1) by Kerr-Newman black holes are even more dramatic than the violations presented in Table 1 which, as emphasized above, are based on the Einstein, Landau-Lifshitz, Papapetrou, and Weinberg prescriptions for calculating energy densities in general relativity [21]

26. J.M. Cohen, F. de Felice, J. Math. Phys. 25, 992 (1984)

27. S.S. Xulu, Mod. Phys. Lett. A 15, 1511 (2000)

28. One therefore concludes that, although Kerr-Newman black holes violate the word of the hoop relation (1) if the mass term $\mathcal{M}$ is interpreted as the mass $M_{\text {in }}\left(r_{+}\right)$contained within the black-hole horizon, these stationary black-hole spacetimes do respect the spirit of the physically important hoop conjecture 